\section{Manejo conservador en una endocarditis infecciosa por Staphylococcus lugdunensis con insuficiencia mitral}

\section{Sr. Director:}

Staphylococcus lugdunensis es un estafilococo coagulasa negativo (ECN) que puede causar endocarditis infecciosa (EI) sobre válvulas nativas, protésicas o marcapasos (1). A diferencia de otros ECN2, la EI por $S$. lugdunensis se caracteriza por una mayor virulencia con la aparición de complicaciones como abscesos perianulares, embolismos sépticos e insuficiencia cardiaca $(1,3-8)$, así como la frecuente necesidad de realizar un recambio valvular precoz (5). Se presenta un caso de EI por S. lugdunensis, que a pesar de lesionar el aparato subvalvular mitral evolucionó favorablemente con tratamiento antibiótico.

Varón de 66 años con antecedentes de enfermedad pulmonar obstructiva crónica (EPOC). Ingresó por cuadro de fiebre, astenia, anorexia y pérdida de $7 \mathrm{~kg}$ de peso de un mes de evolución. La exploración física destacó temperatura axilar de $38^{\circ} \mathrm{C}$ y soplo pansistólico IV/VI en foco mitral. No se apreciaron embolismos periféricos. La analítica mostró: leucocitos $7,7 \times 10^{-3} / \mu 1$ con un $2 \%$ de bandas, hemoglobina $8 \mathrm{~g} / \mathrm{dl}$, plaquetas $52 \times 10^{-3} / \mu \mathrm{l}$ y VSG $>140 \mathrm{~mm} / \mathrm{h}$. La radiografía de tórax fue normal. El ecocardiograma transesofágico evidenció la presencia de una vegetación filiforme en la valva posterior mitral que prolapsaba a aurícula izquierda y un prolapso de la valva anterior causante de una regurgitación de grado moderado-severo. Dos hemocultivos fueron positivos para $S$. lugdunensis resistente a betalactámicos y meticilina. Se inició tratamiento con vancomicina $1 \mathrm{~g} / 12$ horas, rifampicina $600 \mathrm{mg} / 24$ horas y gentamicina $60 \mathrm{mg} / 8$ horas. Ante la naturaleza agresiva del patógeno y la aparición de disfunción valvular se decidió el traslado a un centro de referencia de cirugía cardiaca. No obstante, el paciente evolucionó favorablemente con tratamiento antibiótico durante 6 semanas, sin llegar a precisar cirugía. Durante el seguimiento no presentó insuficiencia cardiaca. A los dos años se le detectó una masa pulmonar con adenopatías. El paciente no volvió a acudir a los controles.

$S$. lugdunensis es un comensal de mucosas y piel, especialmente de la región perineal. Se conoce poco acerca de los factores predisponentes a presentar una infección por este patógeno, aunque se ha constatado que los pacientes suelen tener alguna enfermedad de base como diabetes, insuficiencia renal crónica o neoplasias. En la mayoría de casos se desconoce la puerta de entrada, mientras que en otros se han señalado las infecciones y las manipulaciones quirúrgicas del área perineal (9), así como las infecciones del tracto respiratorio inferior $(5,6)$. Quizás, esto último podría ser el caso de nuestro paciente al ser un EPOC. En una reciente revisión, se ha señalado que esta representa el 1,1\% del total de las EI de una institución, con un $80 \%$ de mortalidad (1). En otra revisión, S. lugdunensis constituía el 18\% de las EI por ECN, y el $44 \%$ de las EI por ECN sobre válvula nativa (3). Puede ser confundida con la EI por $S$. aureus por su capacidad de producir el factor de afinidad por el fibrinógeno (clumping factor) $(2,4)$. Este constituye un factor de virulencia común con $S$. aureus, y es el responsable de la capacidad de positivizar el test de la coagulasa en porta. Se debe tener en cuenta que determinados métodos de laboratorio pueden confundir a $S$. lugdunensis con otros ECN o con $S$. aureus. S. lugdunensis se diferencia del resto de ECN por su positividad en la producción de ornitina descarboxilasa, la prueba de la pirrolidonil-arilamidasa y la acidificación de la manosa $(6,9)$. Ante el hallazgo de hemocultivos positivos para ECN en un caso de EI es necesaria una correcta identificación de la especie, dado que aportará al clínico información útil para el manejo de la misma. Su elevada mortalidad motiva a muchos autores a considerar el tratamiento quirúrgico pre- coz. En la revisión de Anguera y cols., la mortalidad de los pacientes a los que se realizó cirugía fue inferior a los no intervenidos (29 vs. 51\%) (1). Sin embargo, dada la escasez de comunicaciones de EI causadas por este germen, no se conoce que factores pueden predecir una correcta evolución con tratamiento conservador. Existen casos, como el aquí presentado, que a pesar de presentar insuficiencia valvular, e incluso embolismos sépticos, presentaron una correcta evolución sin cirugía $(4,8)$. En cuanto a la sensibilidad antibiótica, la resistencia a meticilina en nuestro medio es de un 7,6\%, mientras que a beta-lactámicos se sitúa entre un 4 y un 7,6\%, alcanzando un $29 \%$ en las series americanas (9).

\section{L. Sorli Redó, A. Aguirre Tejedo ${ }^{1}$, A. Supervía Caparrós ${ }^{1}$, J. L. Echarte Pazos ${ }^{1}$}

Servicios de Medicina Interna y Enfermedades Infecciosas y ${ }^{1}$ Urgencias. Hospital del Mar. Barcelona

1. Anguera I, Del Río A, Miró JM, Martínez-Lacasa X, Marco F, Gumá JR, et al. Staphylococcus lugdunensis infective endocarditis: description of 10 cases and analysis of native valve, prosthetic valve, and pacemaker lead endocarditis clinical profiles. Heart 2005; 91: e10.

2. Aznar R, Miró JM, Pare JC, Gatell JM, Moreno A, Mallolas J, et al. Endocarditis infecciosa por estafilococo coagulasa negativo: revisión de 19 casos. Med Clin (Barc) 1989; 93: 721-6.

3. Patel R, Piper KE, Rouse MS, Uhl JR, Cockerill III FR, Steckelberg JM. Frequency of isolation of Staphylococcus lugdunensis among staphylococcal isolates causing endocarditis: a 20 -year experience. J Clin Microbiol 2000; 38: 4262-3.

4. Wasserman E, Lombard L, Walzl G. Staphylococcus lugdunensis endocarditis in a young, previously healthy female. Eur J Clin Microbiol Infect Dis 1999; 18: 289-91.

5. Renzulli A, Della Corte A, Torella M, Dialetto G, Cotrufo M. Mitral and aortic valve endocarditis due to Staphylococcus lugdunensis. Tex Heart Inst J 2000; 27: 67-9.

6. Van Hoovels L, De Munter P, Colaert J, Surmont I, Van Wijngaerden E, Peetermans W, et al. Three cases of destructive native valve endocarditis caused by Staphylococcus lugdunensis. Eur J Clin Microbiol Infect Dis 2005; $24: 149-52$.

7. Sánchez A, Martínez I, Sanz F, López F, Aguado JM. Endocarditis aguda agresiva por Staphylococcus lugdunensis complicada con múltiples émbolos sépticos cerebrales. Enferm Infecc Microbiol Clin 2000; 18: 526-7.

8. Rodríguez-Gascón M, Roig P, Montagud JB, Merino J. Endocarditis aguda por Staphylococcus lugdunensis con émbolos sépticos cerebrales y pulmonares de buena evolución. Enferm Infecc Microbiol Clin 2003; 21: 463-7.

9. Sánchez P, Buezas V, Maestre JR. Infección por Staphylococcus lugdunensis: presentación de trece casos. Enferm Infecc Microbiol Clin 2001; 19: 475-8.

\section{Hiperplasia nodular regenerativa hepática en una paciente con inmunodeficiencia variable común}

\section{Sr. Director:}

La inmunodeficiencia variable común (IVC) es la inmunodeficiencia primaria más frecuente diagnosticada en adultos. Se diagnostica por la presencia de hipogammaglobulinemia debida a la disminución en los niveles de $\mathrm{IgG}(<500 \mathrm{mg} / \mathrm{dl})$. Habitualmente se acompaña de déficit de Ig A, y en la mitad de los casos también se encuentran bajos los de IgM (1). Clínicamente se caracte- 